\title{
Urethral Undifferentiated Carcinoma
}

National Cancer Institute

\section{Source}

National Cancer Institute. Urethral Undifferentiated Carcinoma. NCI Thesaurus. Code C6168.

An undifferentiated carcinoma arising from the urethra. 